Check for updates

Cite this: Phys. Chem. Chem. Phys., $2019,21,18714$

Received 24th May 2019,

Accepted 7th August 2019

DOI: $10.1039 / c 9 c p 02948 f$

rsc.li/pccp

\title{
Tailoring the mechanical properties of polymer nanocomposites via interfacial engineering $\dagger$
}

\author{
Naishen Gao, ' Guanyi Hou, ${ }^{a}$ Jun Liu, (D) *abcd Jianxiang Shen, ${ }^{e}$ Yangyang Gao, (D) a \\ Alexey $V$. Lyulin (D) $*^{f}$ and Liqun Zhang iD abcdg
}

\begin{abstract}
The improvement of mechanical properties of polymer nanocomposites (PNCs) has been studied for many years, with the main focus on the structure of the nanofillers. Much less effort has been devoted to unraveling the factors controlling the structure of the grafted chains. Herein, through coarse-grained molecular-dynamics simulations, we have successfully fabricated an ideal, mechanically-interlocked composite structure composed of end-functionalized chains grafted to the nanoparticle surface forming rings and making the matrix chains thread through these rings. Depending on the details of the grafting, the reinforcement effect can be remarkable, improving the tensile stress of the system significantly up to $700 \%$. Meanwhile, anisotropy of the system's mechanical response is also observed. Furthermore, the influence of the grafted chain distribution on the mechanical properties of the system has been investigated as well. We observe that the mechanical properties of the system are closely related to the total number of the beads in the grafted chains or the synergistic effect between the length and density of the grafted chains leads to no significant difference in the performance of systems. At constant grafting density, the mechanical properties of the systems correlate negatively to the grafted chain length. In general, our study should help to design and fabricate high-performance PNCs with excellent mechanical properties.
\end{abstract}

\section{Introduction}

Polymer nanocomposites (PNCs) have become a prominent area of current research and development. The addition of hard nanoparticles (NPs) to a polymer matrix leads to a significant improvement of the mechanical, electrical and optical properties of PNCs, ${ }^{1-6}$ signaling that PNCs have high potential value in various applications such as display panels, photonic crystals and memory devices with magnetic nanocomposite multilayers. ${ }^{7-9}$ Over the past

\footnotetext{
${ }^{a}$ Key Laboratory of Beijing City on Preparation and Processing of Novel Polymer Materials, Beijing University of Chemical Technology, People's Republic of China

${ }^{b}$ Beijing Engineering Research Center of Advanced Elastomers, Beijing University of Chemical Technology, People's Republic of China. E-mail: liujun@mail.buct.edu.cn ${ }^{c}$ Engineering Research Center of Elastomer Materials on Energy Conservation and Resources, Beijing University of Chemical Technology, People's Republic of China

${ }^{d}$ Beijing Advanced Innovation Center for Soft Matter Science and Engineering, Beijing University of Chemical Technology, 100029 Beijing, People's Republic of China

${ }^{e}$ College of Materials and Textile Engineering, Jiaxing University, Jiaxing 314001, People's Republic of China

${ }^{f}$ Theory of Polymers and Soft Matter, Department of Applied Physics Technische Universiteit Eindhoven, 5600 MB Eindhoven, The Netherlands.

E-mail:a.v.lyulin@tue.nl

${ }^{g}$ State Key Laboratory of Organic-Inorganic Composites, Beijing University of Chemical Technology, 100029 Beijing, People's Republic of China

$\dagger$ Electronic supplementary information (ESI) available. See DOI: 10.1039/c9cp02948f
}

few decades, many theoretical and experimental studies have revealed that the microscopic morphology of the constituent NPs strongly influences the macroscopic properties of PNCs. ${ }^{10-14}$ Shen et $a .^{15,16}$ investigated the strain-induced non-linear mechanical behavior of a polymer filled with spherical and grafted NPs, and showed that there exist an optimal grafting density and grafted chain length that help to improve the dispersion of the grafted NPs. Moreover, Liu et $a .^{17}$ studied the effect of the filler volume fraction (FVF) and polymer-filler interaction parameters on the mechanical response of the reinforced elastomers, and identified the existence of an optimal FVF for the mechanical reinforcement in the presence of strong polymer-filler interactions. Note that the structure of the polymer chains could have a pronounced effect on the performance of PNCs, by mainly controlling the dispersion state or the spatial distribution of NPs in the polymer matrix. ${ }^{18-22}$ However, it is extremely difficult to produce well-dispersed states of immiscible inorganic NPs in a polymer matrix. At the same time, a good NP dispersion has been shown ${ }^{23}$ to be responsible for good mechanical reinforcement. Various strategies have been suggested for the control of the spatial distribution of NPs, invoking their surface modification such as by adding surfactants (cationic, anionic or nonionic), ${ }^{24-26}$ inorganic surface modification, ${ }^{27,28}$ adsorbent modification, ${ }^{29,30}$ and using grafted polymer chains. ${ }^{29,31}$ The last method is rather popular, ${ }^{32-34}$ and in this case, the grafted chains 
are similar to an amphiphilic material, promoting the compatibility of NPs with the polymer matrix. In this case, the interfacial interactions can be tuned by controlling the grafting density, the grafted chain length, the polymer matrix chain length and the diameter of NPs.

For low grafting densities, the direct aggregation of NPs cannot be fully shielded; in this case, the NPs with uniformly grafted polymer chains will self-assemble into a variety of anisotropic structures ${ }^{18,35-38}$ due to the symmetry breaking of the NP-NP interactions. At sufficiently high grafting density, the grafted polymer chains form "chain brush" conformations, which induce material stretching. Because of the increase of the excluded volume of the grafted NPs, and the enhanced interfacial interaction strength between the grafted chains and the polymer matrix chains, the NPs tend to be dispersed better in the polymer matrix. ${ }^{39-42}$ Besides, the grafted chain length, the strength of the interactions between the grafted chains and the matrix chains, and the effect of the block-copolymer nature of the grafted chains on the dispersion of NPs in the polymer matrix have also been studied systematically. ${ }^{43,44}$

Furthermore, the addition of grafted NPs is also significant for the mechanical properties of the systems. It has been observed that for many PNCs filled with grafted inorganic NPs, such as carbon black or precipitated silica, the Young's modulus and the tensile strength can both decrease by about $40 \%,{ }^{45}$ remain almost unchanged ${ }^{46}$ or increase by about $15 \%,{ }^{47}$ depending on whether the interfacial compatibility between the grafted "brushes" and the polymer matrix is bad, intermediate or good, respectively. However, strong interactions between the grafted "brushes" and the polymer matrix always result in an improvement of the mechanical properties. ${ }^{43}$ Besides, Goyal et al. ${ }^{48}$ employed coarse-grained dynamic simulations to investigate the shear thinning of PNCs filled with grafted NPs, as well as the Brownian stress under uniaxial deformation, suggesting that longer grafted chains could lead to a better reinforcement. The complex viscosity and the storage modulus of the materials increase due to the formation of additional entanglements between the grafted chains and the matrix chains. ${ }^{49}$

The effect of the self-assembled structures of PNCs on the macroscopic properties is important as well. By employing coarse-grained Monte Carlo simulations, Kumar et al. ${ }^{35,50}$ investigated systematically the different self-assembled NP structures with uniformly grafted chains at low grafting densities. They also studied experimentally the corresponding mechanical properties, and observed that the existing sheet structure enhanced the mechanical properties of the PNCs most prominently. Similar results were also obtained by Wang et $a .^{51}$ via self-assembly of polymer-grafted NPs. Using the mesoscale self-consistent field theory and density functional theory approach, the self-assembly of the polymer-grafted NPs was also investigated by Ginzburg, ${ }^{52}$ and similar structures were observed.

In spite of a rather large amount of previous research on the microstructures and the macroscopic properties of PNCs, their mechanical properties still cannot meet the necessary requirements. In particular, mechanical reinforcement is still a great challenge. Recently, Lopez-Moreno et al. ${ }^{53,54}$ studied the reinforcement of polymers by using mechanically interlocked derivatives of single-walled carbon nanotubes, formed by threading the carbon nanotubes through the polymer macrocycles. The improvements of both the Young's modulus and the tensile strength of up to $200 \%$ were observed for the polystyrenenanotube samples with loadings of just $0.01 \mathrm{wt} \%$, signaling that the mechanical bond imparts distinctive advantageous properties to the carbon nanotube derivatives as polymer fillers. The results show that mechanical interlocking is a strategy that can optimize the mechanical performance of polymer composites. Even more so, using bimodal distributed grafted chains is known to be an effective strategy to improve the distribution of NPs and to achieve a good NP dispersion. Schadler et al. ${ }^{6,55,56}$ have shown that particles with monomodal grafted chains easily aggregate, but, fortunately, by mixing sparsely grafted long chains with short grafted chains, the interparticle attraction is screened enough to achieve a good dispersion of NPs, which greatly improves the thermomechanical properties of the nanocomposites. Meanwhile, $\mathrm{Shi}^{57}$ pointed out that rather short grafted chains can not only shield the NP surface from the polymer matrix, but also force the long grafted chains to elongate further into the polymer matrix.

In this study, by using coarse-grained molecular-dynamics simulations (CGMDS), ${ }^{58}$ we investigate the relationship between the structure and the mechanical properties of a mechanically interlocked polymer system via grafting the polymer chains to the NP surface with both ends, and making ring structures. Ungrafted matrix chains have to thread through these grafted rings, and entangle with them. We study the effects of the stiffness of the matrix chains and the ring shape of the grafted chains on the formation of this mechanically interlocked structure. We also simulate and analyze the influence of the bimodal distribution of the grafted polymers on the composite mechanical properties, in the hope of providing scientific guidance for the design and fabrication of PNCs with remarkable and adjustable mechanical properties tailored for practical applications.

\section{Model and simulation method}

We adopted the classical CGMDS to study the mechanical properties of the interlocked and bimodal polymer-grafted nanocomposites. The polymer chains were represented by the bead-spring model developed by Kremer and Grest. ${ }^{59}$ The monomers were lumped together into spherical beads, and the beads were connected by elastic springs. ${ }^{60}$ Here, since it is not our target to study a specific polymer, we use reduced units, in which $\varepsilon$ is the LJ energy parameter, and $m$ and $\sigma$ are the mass and diameter of the monomer, respectively. This means that all calculated quantities are dimensionless. One polymer bead with a diameter of $\sigma$ (using the Lennard-Jones length unit) corresponds to 3-6 monomers in a realistic polymer chain such as, for example, polybutadiene. ${ }^{61-63}$ A simulated matrix polymer chain consisted of $L_{\mathrm{m}}=100$ beads with mass $m$ and diameter $\sigma$. The number of the matrix polymer chains is $N_{\mathrm{m}}=100 \mathrm{in}$ both the mechanically interlocked and bimodal systems, as explained 
later. The bead size of the grafted chains is equal to that in the matrix chains. Nevertheless, we stress that in reality, the grafted polymers can be chemically different from the matrix.

The nanoparticles were represented by beads with a diameter of $4 \sigma$ and a mass of $64 \mathrm{~m}$. Two systems, one with the mechanically interlocked structure and another one with NPs modified by the bimodal distributed grafted polymer chains, have been simulated with 30 NPs. In the two systems, the grafted chains were randomly distributed on the surface of NPs, using the chemical bond between the head atom of the grafted chain and the NP. The exact position of the contact can slip on the NP surface during the equilibration and stretching process. The total number of grafted polymer chains is characterized by the grafting density $\Sigma$ as

$$
\Sigma=\frac{Q}{4 \pi R_{\mathrm{NP}^{2}}}
$$

where $Q$ is the number of grafted sites per NP, and $R_{\mathrm{NP}}$ is the NP radius.

The non-bonded interactions between all the beads, including polymer-polymer, polymer-nanoparticle, and nanoparticle-nanoparticle, were described by the truncated and shifted Lennard-Jones (LJ) potential

$$
U(r)= \begin{cases}4 \varepsilon\left[\left(\frac{\sigma}{r-\Delta}\right)^{12}-\left(\frac{\sigma}{r-\Delta}\right)^{6}\right]+C & r<r_{\text {cutoff }}+\Delta \\ 0 & r \geq r_{\text {cutoff }}+\Delta\end{cases}
$$

where $\varepsilon$ is the pair interaction energy parameter, $r$ is the distance between two interaction sites, and $\sigma$ is the length unit. $\Delta$ is a distance unit, taking into account the effect of the excluded volume of the different interaction sites, which depends on the size of polymer beads and NPs. For polymerNP and NP-NP interactions, $\Delta$ is chosen to be $R_{\mathrm{NP}}-\sigma / 2$ and $2 R_{\mathrm{NP}}-\sigma$, respectively, and for polymer-polymer interaction, $\Delta$ becomes zero. $C$ is a constant that guarantees the continuity of the potential energy at the cutoff distance. Hence, the actual cutoff distance of the implemented potential is the sum of $r_{\text {cutoff }}$ and $\Delta$. The LJ potential was cut off at different distances to model attractive or repulsive interactions. The repulsive interactions were simulated by setting $r_{\text {cutoff }}=2^{1 / 6} \sigma$, whereas $r_{\text {cutoff }}=2 \times 2^{1 / 6} \sigma$ and $r_{\text {cutoff }}=2.5 \sigma$ represented a simulated short-ranged attraction and a long-ranged attraction, respectively.

The bond interactions between the adjacent beads were modeled by a harmonic potential, ${ }^{64}$

$$
U_{\text {bond }}=\frac{1}{2} K\left(r-r_{0}\right)^{2}
$$

where $K$ is a bond strength constant, $K=200$, and $r_{0}$ is the equilibrium bond distance. The harmonic potential was proved to be efficient in modeling polymer chains. ${ }^{65-68} r_{0}$ between adjacent beads in one polymer chain is equal to 1.0 , and $r_{0}$ between the polymer and NP is equal to 2.5.
The bending angle of the polymer chain between three consecutive beads was modeled by a harmonic potential, ${ }^{69}$

$$
U_{\text {angle }}=\frac{1}{2} k\left(\theta-\theta_{0}\right)^{2}
$$

where $k$ is a constant, varied to simulate polymer chains with different stiffness, and $\theta_{0}$ is the equilibrium value of the angle. Upon increasing the value of $k$ (ranging from 0 to 30 in the present simulations, with $k$ equal to 0 for the most flexible polymer chains), the chain stiffness increases correspondingly. This, in turn, reduces the entanglement length. ${ }^{60,70-72}$

In the present simulations of the mechanically interlocked system (the first simulated model), the grafted polymer chains were made to be bi-functional at both ends. To create the grafted ring structure, it was necessary to graft the two ends of a grafted chain onto one NP, so that the grafted rings and the remaining matrix chains can form the mechanically interlocked structure, the grafted-ring system (GR system), easily, as shown in Fig. 1(a). In the presented simulations, we have fixed the grafting density to $\Sigma=0.2$, and the length of all the grafted chains was equal to $L_{\mathrm{g}}=25$. Initially, the simulated box contained only three NPs. This initial box was replicated in both $X$ and $Y$ directions, resulting in a 10 times larger system.

The challenge at this stage was to make a mechanically interlocked structure, and there were two key points in the system construction process that can be mentioned: one has to let the matrix chains thread through the ring, and, after that, one has to lock up the mechanically interlocked structure. To achieve these two purposes, the whole equilibration process was carried out in two steps. During the first equilibration step, the matrix-matrix and the graft-graft LJ interactions were set to be repulsive. This helps to have both the matrix chains and the grafted rings stretched enough, which is beneficial for the matrix chains to thread through the grafted rings. In the second equilibration step, both matrix-matrix and graft-graft interactions were set to be attractive, which is beneficial to lock up the already interlocked structure. The details of all the LJ interaction parameters during the equilibration are listed in Table 1. As our main goal is to provide some modelling insights into the influence of the mechanically interlocked structure on the mechanical properties of PNCs, we compared the tensile properties of the simulated grafted-ring composite system (GR system) with those of the grafted-linear composite system (GL system), as shown in Fig. 1(a), under the same other conditions.

In the second simulated model, the grafted polymer chains of the same structure (functional at only one end), but with different length, were grafted onto each NP. In what follows, this system will be referred to as a "Bimodal polymer-grafted NP". The length of the grafted short chains was fixed to $L_{\mathrm{S}}=25$, and the grafted long chain length $L_{1}$ was varied from $L_{1}=25$ to $L_{1}=100$. Each grafted chain had only one grafting site at the end that would be bonded to the NP surface during the grafting process, and the formation of the ring structure was prevented. Thirty of such bimodal polymer-grafted NPs were then mixed homogeneously with the matrix chains $\left(L_{\mathrm{m}}=100\right.$ and $\left.N_{\mathrm{m}}=100\right)$, as shown schematically in Fig. 1(b). 


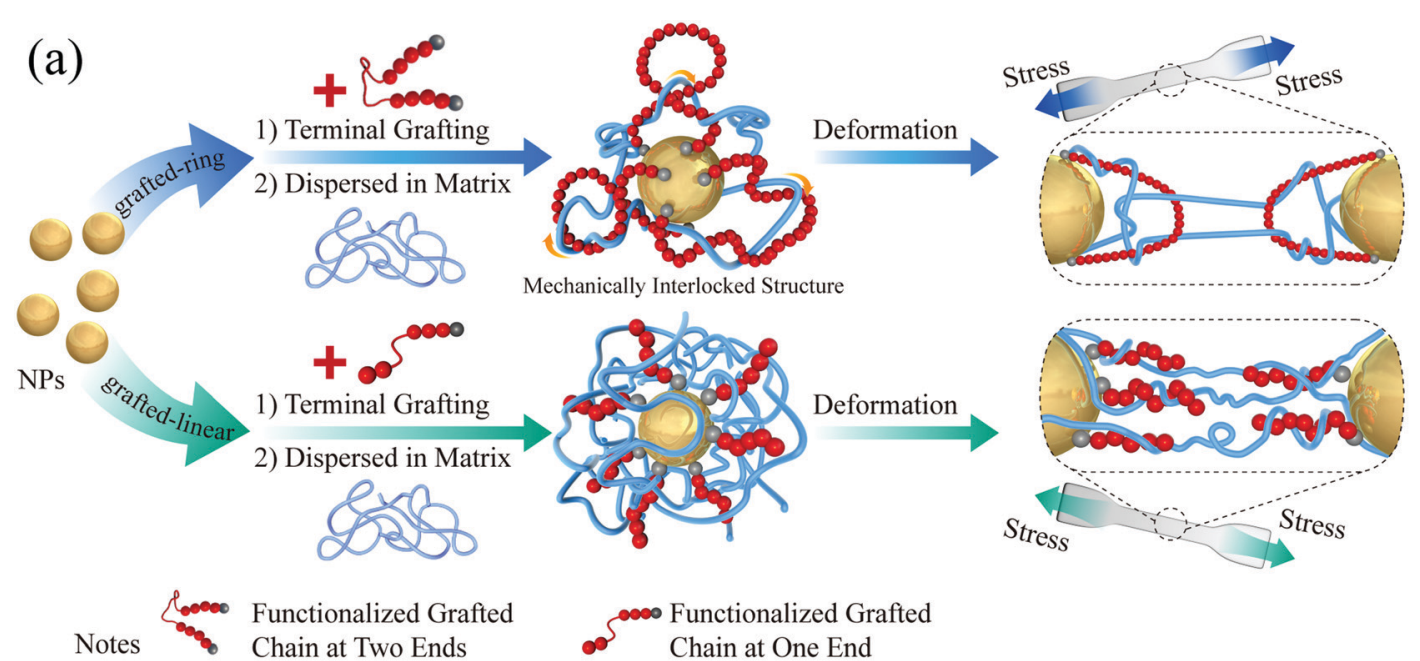

Chain at One End

(b)
Grafted Long Chains

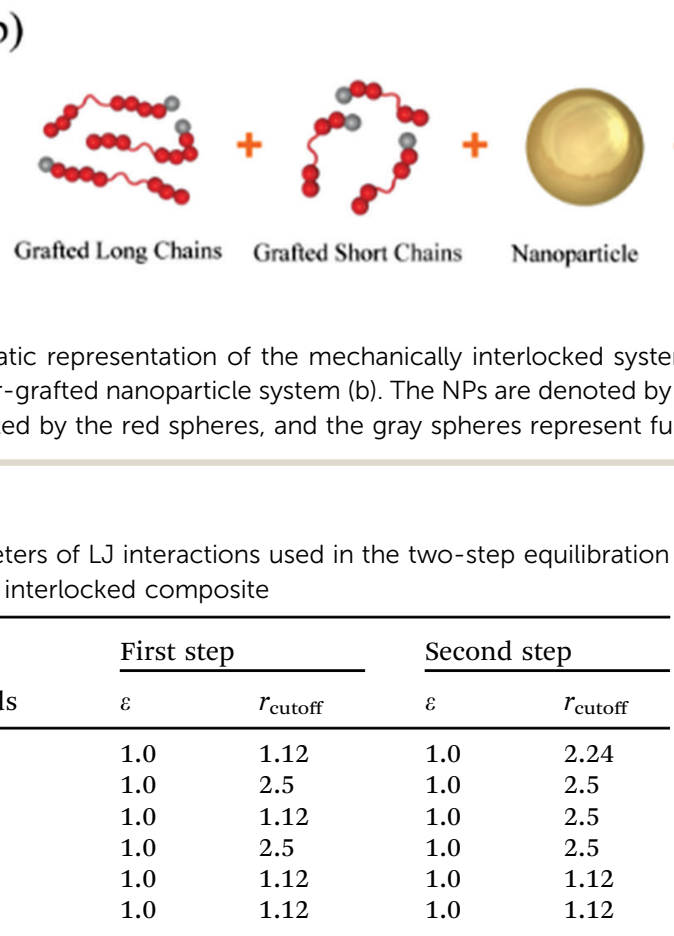

The fraction of the grafted chains $\left(C_{\mathrm{g}}\right)$ was determined as:

$$
C_{\mathrm{g}}=\frac{n_{\mathrm{g}}}{n_{\mathrm{g}}+n_{\mathrm{m}}}
$$

where $n_{\mathrm{g}}$ is the total number of grafted chain beads, $n_{\mathrm{g}}=L_{\mathrm{l}} \times$ $N_{\mathrm{l}} \times 30+L_{\mathrm{s}} \times N_{\mathrm{s}} \times 30$, and $n_{\mathrm{m}}$ is the total number of beads of the matrix chains, $n_{\mathrm{m}}=L_{\mathrm{m}} \times N_{\mathrm{m}}$, where $N_{\mathrm{l}}$ and $N_{\mathrm{s}}$ represent the number of long chains and short chains grafted on each NP, respectively. Here, we explored two cases: one corresponded to $C_{\mathrm{g}}=0.6$, and the other corresponded to $\Sigma=0.4\left(C_{\mathrm{g}}\right.$ varied from 0.6 to 0.79 ). In the former case, $C_{\mathrm{g}}$ is fixed ( $n_{\mathrm{g}}$ and $n_{\mathrm{m}}$ are fixed), so the increasing of the grafted long chain length $\left(L_{1}\right)$ inevitably leads to a smaller amount of grafted chains; the details are listed in Table 2. We try to keep the number of long and short chains grafted on each NP equal to each other. For the latter, we fix the grafting density to $\Sigma=0.4, N_{\mathrm{l}}=10$ and $N_{\mathrm{S}}=10$.
Table 2 Parameters of the simulated PNCs with $C_{g}=0.6^{a}$

\begin{tabular}{lllll}
\hline System & $N_{\mathrm{l}} / N_{\mathrm{s}}$ & $L_{\mathrm{l}} / L_{\mathrm{s}}$ & $n_{\mathrm{g}} / n_{\mathrm{m}}$ & $C_{\mathrm{g}}$ \\
\hline 1 & $10 / 10$ & $25 / 25$ & $15000 / 10000$ & 0.6 \\
2 & $7 / 6$ & $50 / 25$ & $15000 / 10000$ & 0.6 \\
3 & $5 / 5$ & $75 / 25$ & $15000 / 10000$ & 0.6 \\
4 & $4 / 4$ & $100 / 25$ & $15000 / 10000$ & 0.6 \\
\multicolumn{5}{c}{${ }^{a} N_{\mathrm{l}}$ - number of grafted long chains per NP, $N_{\mathrm{s}}-$ number of grafted } \\
short chains per NP, $L_{\mathrm{l}}-$ length of the grafted long chains, $L_{\mathrm{s}}-$ length of \\
the grafted short chains, $n_{\mathrm{g}}-$ total number of grafted beads, $n_{\mathrm{m}}-$ total \\
number of matrix beads, $C_{\mathrm{g}}-$ the fraction of grafted chains.
\end{tabular}

Upon increasing $L_{\mathrm{l}}, C_{\mathrm{g}}$ increases correspondingly; the composition details are listed in Table 3.

In the simulations with "Bimodal polymer-grafted NP" systems, both grafted chains and the matrix chains were flexible and

Table 3 Parameters of the simulated PNCs with increasing $C_{\mathrm{g}}{ }^{a}$

\begin{tabular}{lllll}
\hline System & $N_{\mathrm{l}} / N_{\mathrm{s}}$ & $L_{\mathrm{l}} / L_{\mathrm{s}}$ & $n_{\mathrm{g}} / n_{\mathrm{m}}$ & $C_{\mathrm{g}}$ \\
\hline 1 & $10 / 10$ & $25 / 25$ & $15000 / 10000$ & 0.6 \\
2 & $10 / 10$ & $40 / 25$ & $19500 / 10000$ & 0.66 \\
3 & $10 / 10$ & $50 / 25$ & $22500 / 10000$ & 0.69 \\
4 & $10 / 10$ & $75 / 25$ & $30000 / 10000$ & 0.75 \\
5 & $10 / 10$ & $100 / 25$ & $37500 / 10000$ & 0.79
\end{tabular}

${ }^{a} N_{\mathrm{l}}$ - number of grafted long chains per NP, $N_{\mathrm{s}}$ - number of grafted short chains per NP, $L_{1}$ - length of the grafted long chains, $L_{\mathrm{s}}$ - length of the grafted short chains, $n_{\mathrm{g}}$ - total number of grafted beads, $n_{\mathrm{m}}$ - total number of matrix beads, and $C_{\mathrm{g}}$ - the fraction of grafted chains. 
corresponded to chemically different polymers. We, nevertheless, set the interaction energy parameter $\varepsilon$ between beads corresponding to matrix-matrix $\varepsilon_{\mathrm{mm}}$, grafted-grafted $\varepsilon_{\mathrm{gg}}$, matrix-grafted $\varepsilon_{\mathrm{mg}}$, matrix-NP $\varepsilon_{\mathrm{mn}}$, grafted-NP $\varepsilon_{\mathrm{gn}}$, and NPNP $\varepsilon_{\mathrm{nn}}$ interactions all equal to 1.0. To avoid agglomeration of the grafted chains, repulsive interactions between the graftedgrafted polymer chains were introduced by setting $r_{\text {cutoff }}=2^{1 / 6} \sigma$. The attractive interactions between the matrix-grafted chains were modelled by setting $r_{\text {cutoff }}=2.5 \sigma$ to promote entanglements. Weak attractions between the matrix-matrix chains were introduced by setting $r_{\text {cutoff }}=2 \times 2^{1 / 6} \sigma$, whereas the NPNP parameter was set to be $r_{\text {cutoff }}=2^{1 / 6} \sigma$ to help dispersion.

The simulations were started from a non-overlapped configuration of all matrix chains and polymer-grafted NPs in a large simulation box. The $N P T$ and $N V T$ ensembles were adopted, where the temperature was fixed at $T^{*}=1.0$, well above the glass-transition temperature for all the simulated systems. The pressure was set at $P^{*}=1.0$. The Nose-Hoover thermostat and barostat were used for temperature and pressure coupling. Periodic boundary conditions were applied in all three directions. The equations of motion were integrated using the velocity-Verlet algorithm with a time step $\Delta t=$ $0.001 \tau$, where $\tau$ is the LJ time unit $\tau=\sigma \sqrt{\frac{m}{\varepsilon}}$. The equilibration process was performed over a long time, for more than $1 \times 10^{7}$ time steps for each system, to ensure that each polymer chain had moved at least $2 R_{\mathrm{g}}$, where $R_{\mathrm{g}}$ is the corresponding rootmean-square radius of gyration.

After sufficient equilibration, the uniaxial tensile deformation was performed to obtain the stress-strain characteristics, similar to the approach utilized in our previous studies. ${ }^{73,74}$ The box length in the $X$ direction was increased at a constant engineering strain rate, while the box lengths in the $Y$ and $Z$ directions were reduced simultaneously so as to maintain constant box volume. The strain rate was specified as $\dot{\varepsilon}=\Delta L / \Delta t=\frac{L(t)_{X}-L_{X}}{L_{X}} / \Delta t=0.0327 / \tau$. The average stress $\sigma$ in the $X$ direction was obtained from the deviatoric part of the stress tensor $\sigma=(1+\mu)\left(-P_{X X}+P\right) \approx 3\left(-P_{X X}+P\right) / 2$, where $P=\sum_{i} P_{i i} / 3$ was the hydrostatic pressure, taking stretching in the $X$ direction as an example. The parameter $\mu$ stands for the Poisson's ratio, which was equal to 0.5 in the present simulations. Here, we conducted uniaxial tensile deformation in the $X, Y$ and $Z$ directions for mechanically interlocked systems, respectively, to characterize the mechanical anisotropy. Although the anisotropy of mechanical properties has been observed for even larger simulated samples, polymer systems of much larger size are required to make definite predictions about the mechanical anisotropy on the macroscopic scale. However, since the grafted chains and the matrix chains are both flexible, the system has an isotropic stressstrain behavior, which has been confirmed. Only the uniaxial tensile deformation in the $X$ direction was performed for the bimodal polymer-grafted NP systems.
All the production runs were carried out by using the large-scale atomic/molecular massively parallel simulator (LAMMPS) software developed by Sandia National Laboratories. ${ }^{75}$ More simulation details can be found in our previous publications. ${ }^{15,76}$

\section{Results and discussion}

\subsection{Mechanically interlocked system}

3.1.1 Effect of the matrix chain stiffness. In the mechanically interlocked system, the grafted chains are set to be relatively
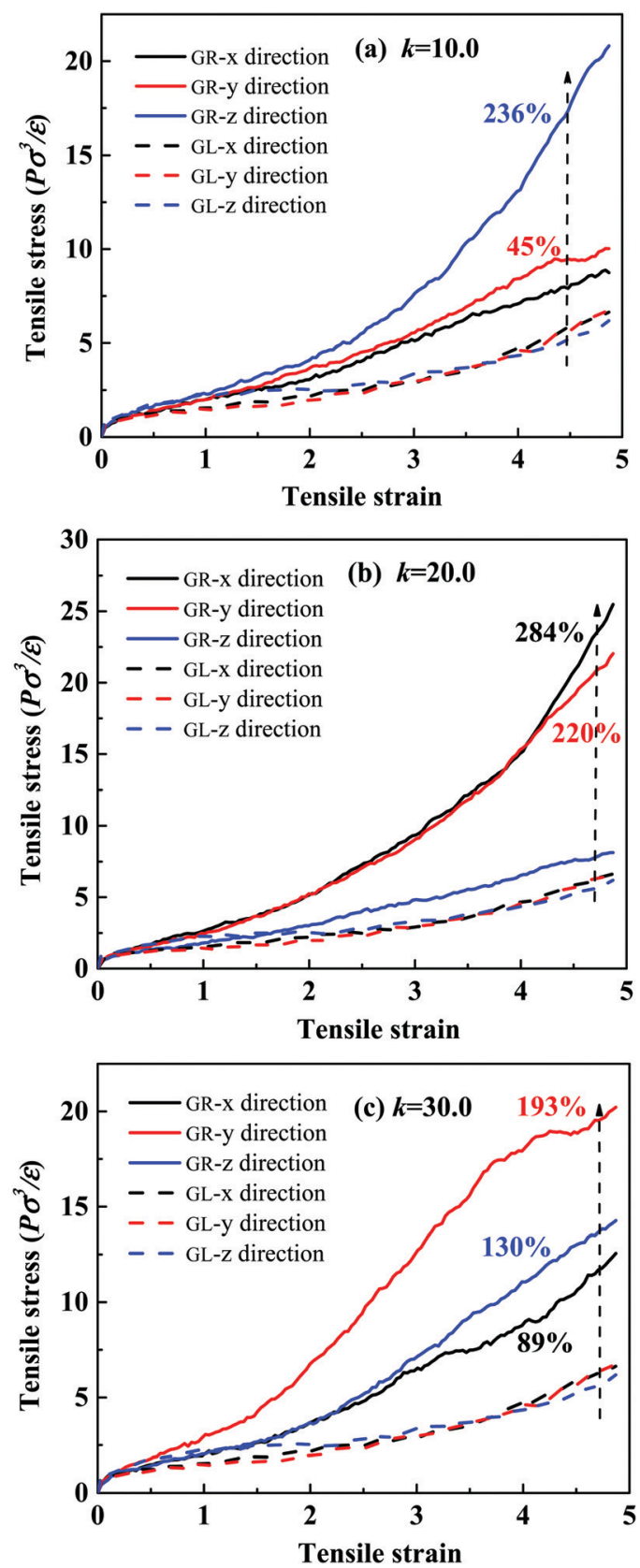

Fig. 2 Stress-strain behavior of the grafted-ring (GR) system and the graftedlinear (GL) system in three deformation directions with different stiffness $k$ values of matrix chains in the first equilibration step: (a) $k=10.0$, (b) $k=20.0$, and (c) $k=30.0$. Black, red and blue colors represent $X, Y$ and $Z$ directions, respectively. 
stiff $\left(k=20.0\right.$ and $\theta_{0}=180.0$, see eqn (4)) to allow matrix chains to thread through the rings easily. The matrix stiffness effects on the formation of the mechanically interlocked structure have been analyzed. Through previous attempts, we know that whether the matrix chains are flexible or stiff is not conducive to the formation of an ideal mechanically interlocked structure; more detailed analysis is shown in S1 and S2 (ESI $\dagger$ ). Therefore, we envisage a new method wherein the matrix chains are all set as stiff in the first equilibration process to facilitate the matrix chains threading through the rings, and after that, they are set as flexible in the second equilibration process to facilitate locking of the structure obtained in the first step. We adjust the value of $k$ of the matrix chains in the first equilibration step to be 10.0, 20.0, and 30.0. After the equilibration, the tensile properties of the systems were investigated, see Fig. 2. The percentages of different colors represent the stress increment in the corresponding direction. The amount of stress increase was calculated in the GR system relative to the GL system in the corresponding direction (calculated by using the stress at the maximum strain). It is obvious that compared to the GL system, the tensile properties of the GR system have been improved in two or even three directions. The enhancement effect is the most significant at $k=20.0$, and reaches up to $300 \%$ and $220 \%$ in the $X$-direction and $Y$-direction, respectively. Namely, this method can effectively improve the mechanical properties of the system.

To understand the reason for the significant enhancement of the mechanical properties, we probe the microscopic morphology of the system. Taking $k=10.0$ as an example, the typical snapshots of each step are shown in Fig. 3(a), highlighting one $\mathrm{NP}$, its grafted rings, and five matrix chains entangled with the grafted rings. After the first step of equilibration, the matrix chains are arranged along the contour of the rings due to the attraction interactions. Moreover, penetration, or threading of the matrix chains into the grafted rings, is also observed. A slight agglomeration of the matrix chains occurs after the second step of equilibration, but, importantly, the threading of the matrix chains through the rings is not affected. From the analysis of the snapshots, we conclude that the desired mechanically interlocked structure is formed.

Next, we study the morphological evolution of the system during the uniaxial deformation (the $Z$ direction was chosen), and the snapshots are presented in Fig. 3(b). For clarity, only one grafted-ring NP and five typical matrix chains are shown. We see that the conformation of the polymer chains in the
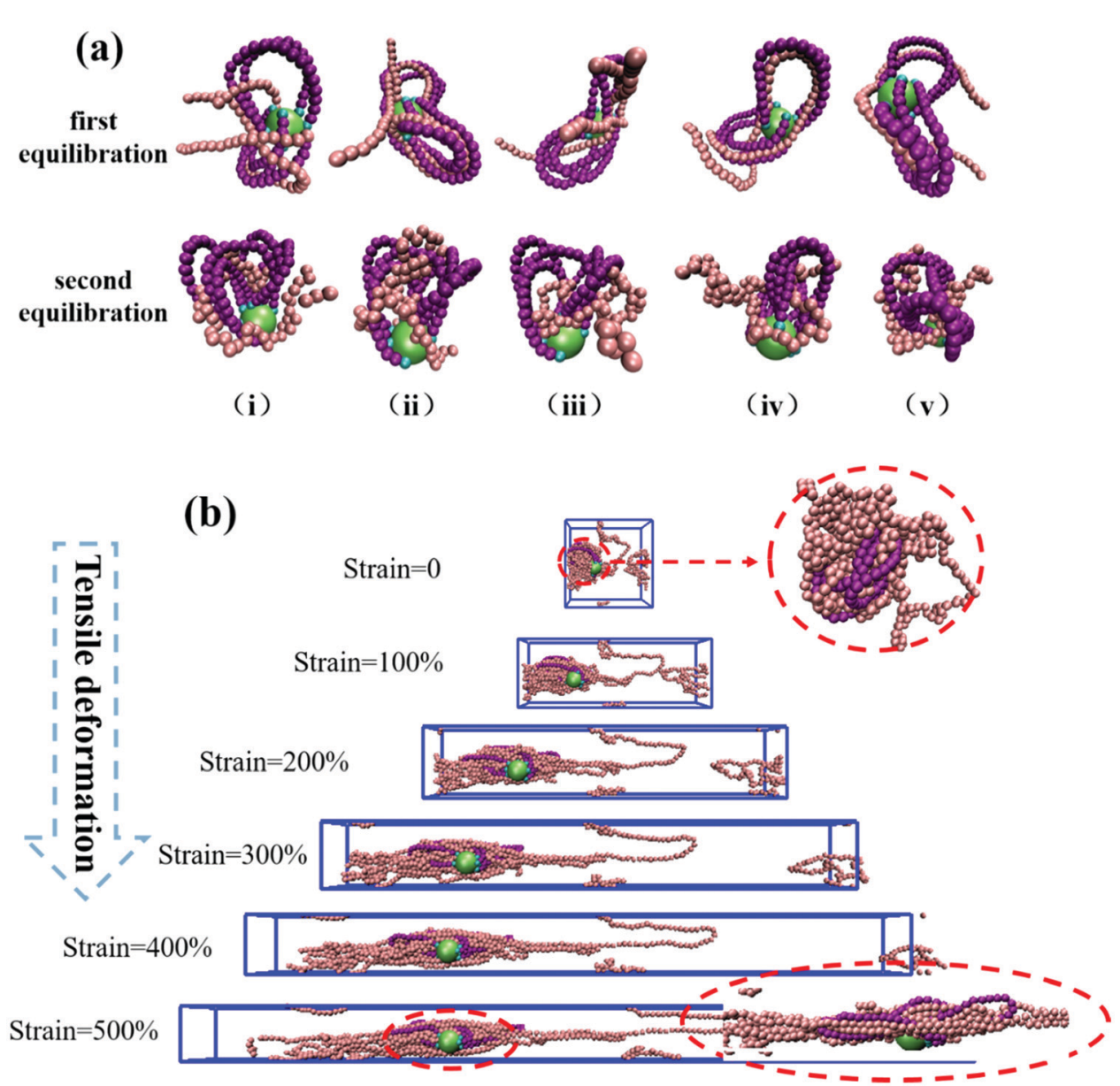

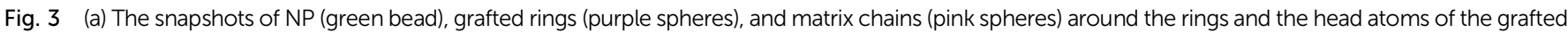

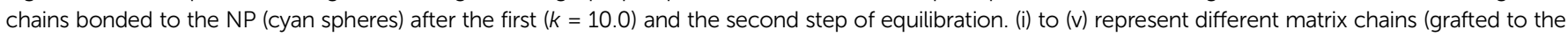
same NP) threading through the rings. (b) Snapshots of the uniaxially deformed structures, circles on the right enlarge parts of the deformed system. 
deformation direction becomes extended, while in the $X$ and $Y$ directions, the conformations become significantly folded. Surprisingly, the matrix chains and the grafted rings are still entangled very tightly at an extremely large strain of $500 \%$, which can explain the significant stress increase in the deformation direction. Some matrix chains do not entangle so tightly, and they tend to slip out of the rings during the stretching process, causing non-uniformity of the chain elongation and anisotropic stress-strain behavior, as clearly seen in Fig. 2.

In addition to these intuitive snapshots reflecting the microscopic structure, there are other characterization methods that can be used to explain the microscopic mechanism for the mechanical enhancement. There is no doubt that the existence of a mechanically interlocked structure will inevitably promote the entanglement and agglomeration of polymer chains, which can be reflected by the mean-square radius of gyration $\left(R_{\mathrm{g}}{ }^{2}\right)$. By comparing $R_{\mathrm{g}}{ }^{2}$ of the matrix chains in the GR system and GL system during the uniaxial deformation, shown in Fig. 4(a), we observe that $R_{\mathrm{g}}{ }^{2}$ of the matrix chains in the GR system is lower than in the GL system, indicating that the matrix chains in the GR system are more agglomerated. Since the agglomeration of polymer chains may affect the interaction of the system, the non-bonded interaction energy ( $\left.U_{\text {non-bonded}}\right)$ was characterized for different bead pairs of the GR system and GL system as a function of the tensile strain. It is important to note that the smaller (negative) the nonbonded interaction energy, the stronger the interactions between the corresponding parts. Obviously, as can be seen in Fig. 4(b), the strength of the non-bonded interactions of matrix-graft and matrix-matrix in the GL system decrease sharply after 300\% strain, resulting in a dramatic total non-bonded interaction decrease. The non-bonded interactions of matrix-graft and matrix-matrix in the GR system change very little during the whole deformation process, indicating that the mechanically interlocked structure not only effectively enhances the interfacial interaction, but also promotes the entanglement between the matrix chains, thereby improving its mechanical properties.

What's more, to provide further understanding on the stress-strain behavior, we quantitatively calculate the distribution of different components on the total tensile stress in the GR system and the GL system, as shown in Fig. 4(c and d). The red curve represents the total tensile stress. The green, magenta and blue curves represent the stress component of the polymer matrix, grafted chains and NPs, respectively. By analyzing Fig. 4(c and d), it is clear that the mechanically interlocked structure can significantly enhance the mechanical properties of a system mainly by increasing the stress of the matrix chains during the stretching process, caused by the entanglement of matrix-matrix and matrix-graft.
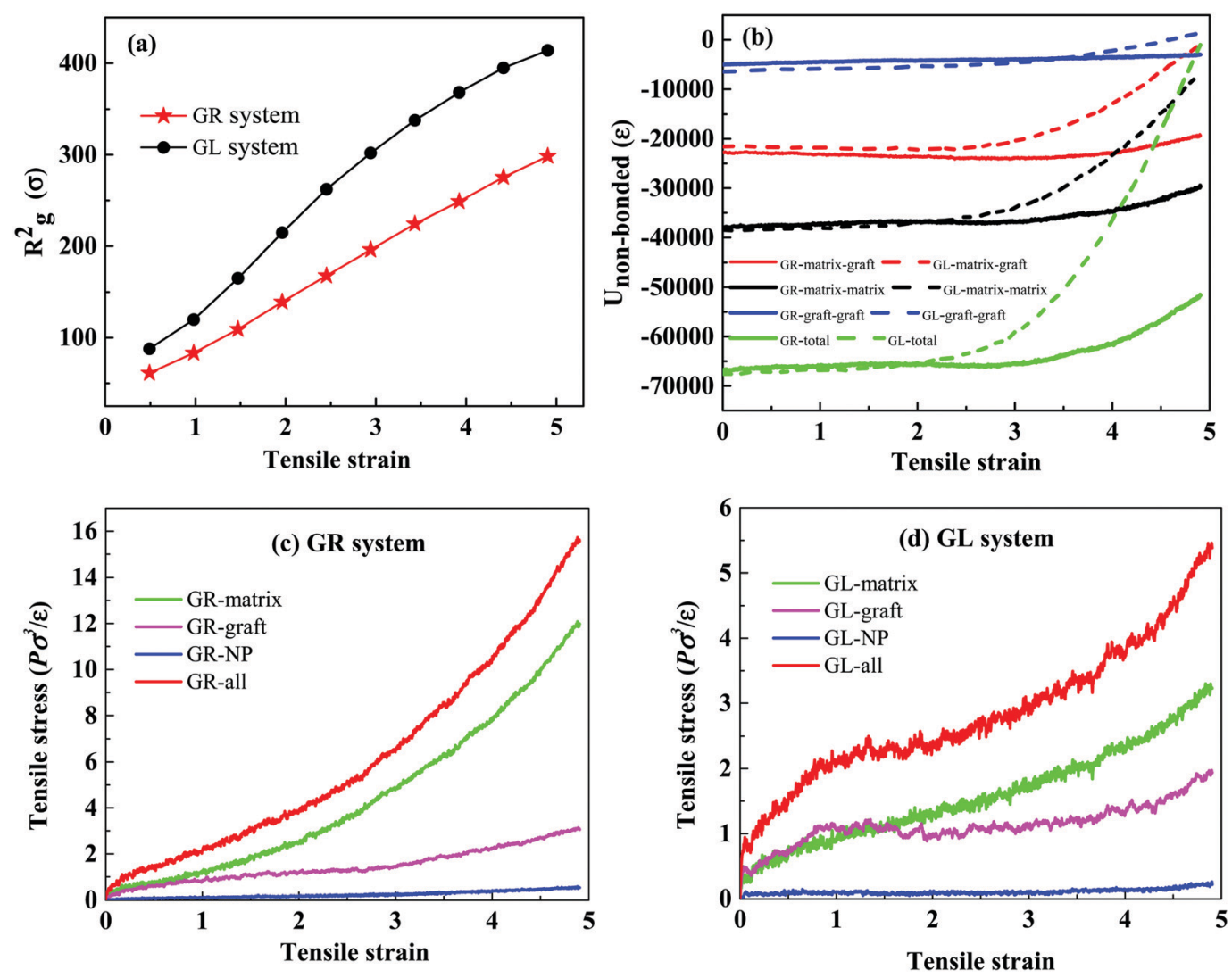

Fig. 4 (a) The mean-square radius of gyration $R_{\mathrm{g}}{ }^{2}$ of the matrix chains in GR and GL systems during the uniaxial deformation. (b) The non-bonded interaction energy of all parts of the grafted-ring (GR) system and the grafted-linear (GL) system, during the uniaxial deformation. The stress distributions of different components of the total tensile stress in the GR system (c) and the GL system (d). The, green, magenta, blue and red curves represent the stress components of the polymer matrix, grafted chains, NPs and the total tensile stress, respectively. 
3.1.2 Effect of the grafted ring structure. The structure of the grafted rings has been changed by adjusting the equilibrium value of the valence bending angle, $\theta_{0}=60.0,120.0$, and 180.0, keeping the stiffness $k$ constant. With the change of $\theta_{0}$, the architecture and the spatial dimensions of the grafted rings also change accordingly. As shown in Fig. S3 (ESI $\dagger$ ), when $\theta_{0}=180.0$, the rings are large and smooth, and interpenetration between the rings is also observed. As the value of $\theta_{0}$ decreases, the rings become distorted, and their size decreases. Thus, the value of $\theta_{0}$ plays a decisive role in the structure of the rings, as well as in the formation of the mechanically interlocked structures. The effect of changing the value of $\theta_{0}$ on the
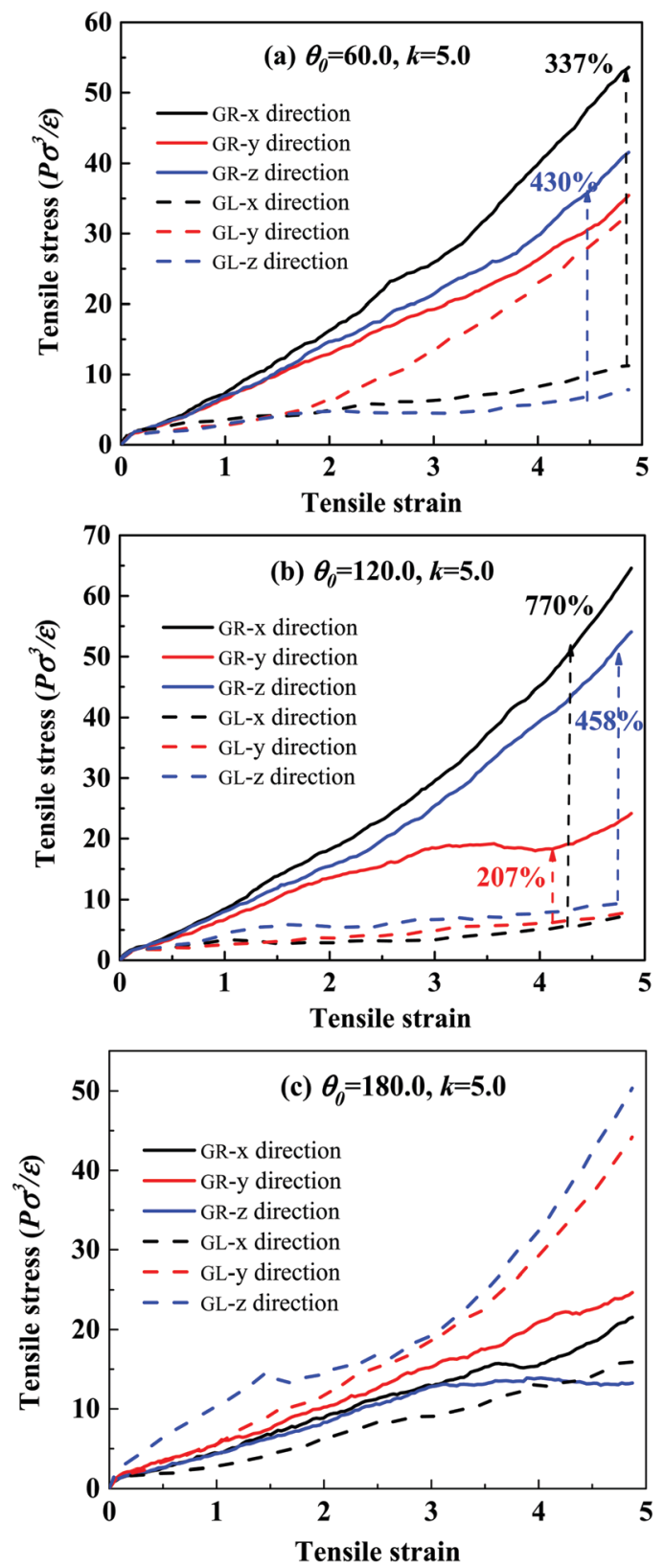

Fig. 5 Stress-strain behavior of the grafted-ring (GR) system and the grafted-linear (GL) system in three deformation directions with different $\theta_{0}$ values. Black, red and blue colors represent $X, Y$ and $Z$ directions, respectively. mechanical properties of the composites is shown in Fig. 5 for $k=5.0$ of the matrix chains. The stress enhancement is obvious at $\theta_{0}=60.0$, it is prominent at $\theta_{0}=120.0$, and it is rather unnoticeable at $\theta_{0}=180.0$. Such a non-monotonic stress enhancement is attributed to the difference of the ring structures caused by the choice of $\theta_{0}$. When $\theta_{0}$ is too small, the rings do not have enough space for matrix chains to thread; when $\theta_{0}$ is too big, the size of the rings is too large, causing the matrix chains to slip out of the rings easily during the deformation. As for $\theta_{0}=120.0$, the rings are apparently optimal for matrix chains to thread, and the graft-matrix structure can be retained to provide a great improvement of the mechanical properties. In particular, the strongest enhancement of mechanical properties appears when $k=5.0$ and $\theta_{0}=120.0$, reaching up to $770 \%$, $458 \%$ and $207 \%$ in the $X, Y$, and $Z$ directions, respectively. This is expected to provide some scientific guidelines to fabricate novel PNCs with excellent mechanical properties. At the same time, anisotropic stress-strain behavior is observed in both the grafted-ring (GR) system and the grafted-linear (GL) system. One of the reasons for this is the non-uniformity of the chain elongation because of the mechanically interlocked structure. The other is the uneven orientation of the matrix chains due to the stiffness and the mutual attraction. (i)

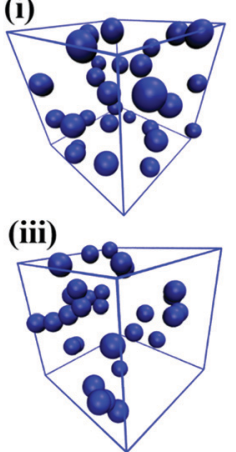

(ii)

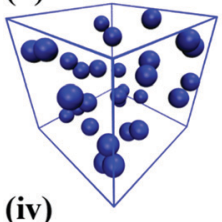

(iv)

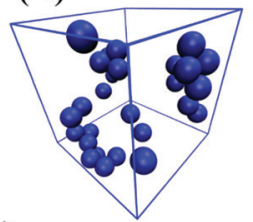

(a)

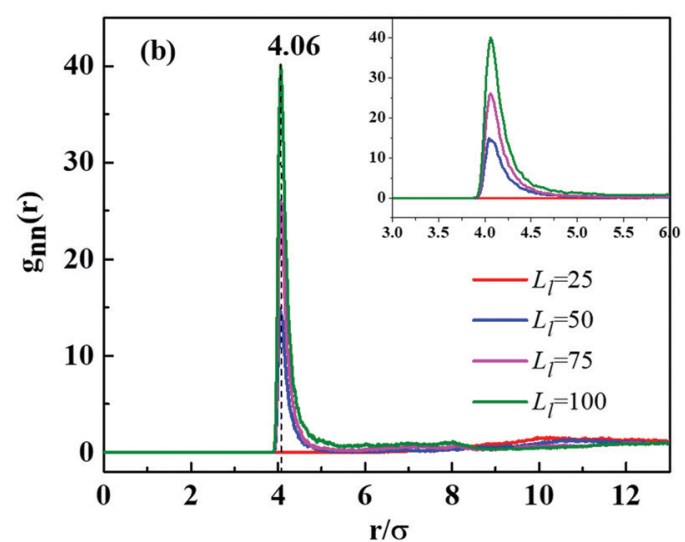

Fig. 6 (a) The snapshots of NPs corresponding to different grafted long chain length $L_{1}$ at $C_{g}=0.6$ : (i) $L_{1}=25$, (ii) $L_{1}=50$, (iii) $L_{1}=75$ and (iv) $L_{1}=100$. The blue spheres denote NP cores. For clarity, polymer chains are not shown. (b) Radial distribution function (RDF) of NP-NP with different $L_{1}$. The inset is introduced to clearly show the different values of the three peaks. 


\subsection{Bimodal polymer-grafted NP system}

3.2.1 Effect of bimodal distribution of grafted chains at $\boldsymbol{C}_{\mathbf{g}}=\mathbf{0 . 6}$. To investigate the effect of the grafted chain length on the mechanical properties of the polymer-grafted NP system, we designed a set of experiments, as listed in Table 2. The snapshots for the dispersion states of NPs with different $L_{1}$ values in the polymer matrix are shown in Fig. 6(a). We observe that the
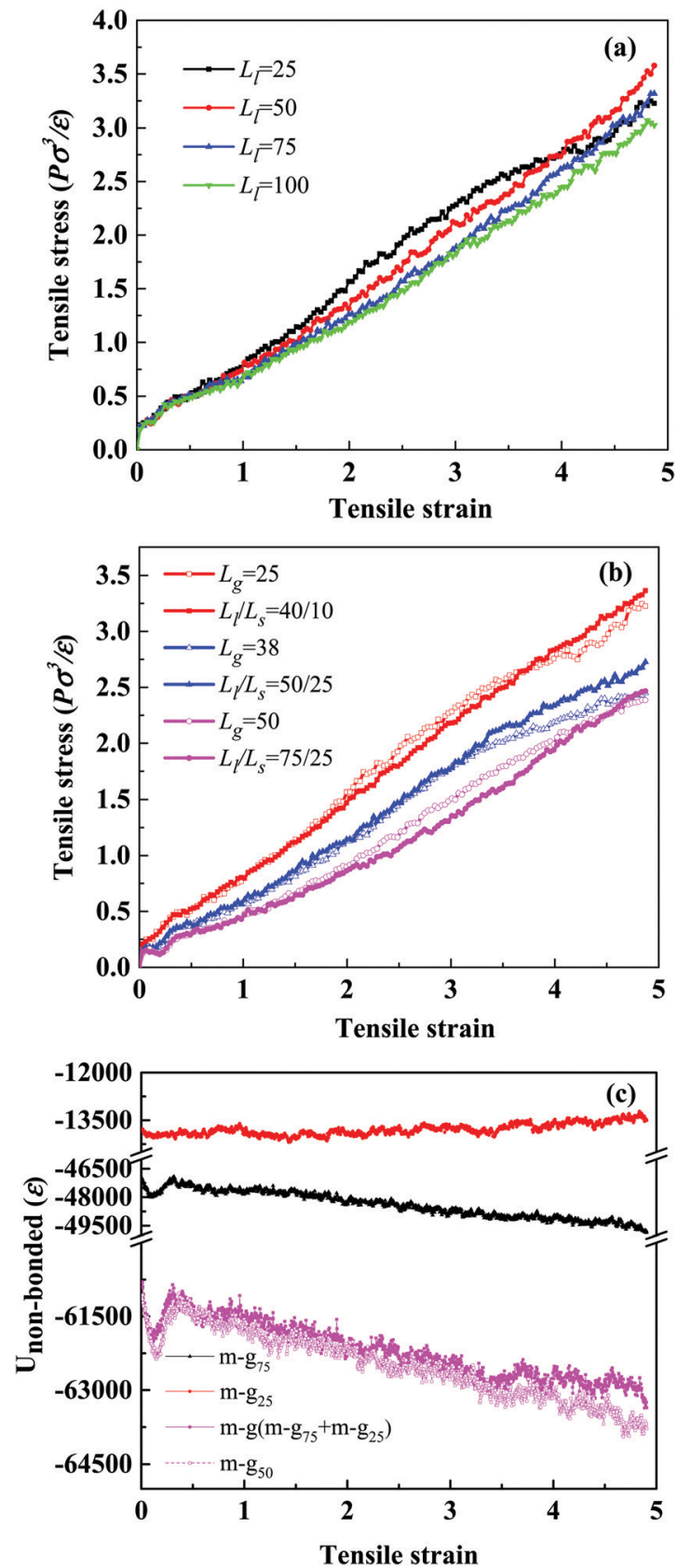

Fig. 7 (a) The stress-strain curves of simulated systems with different $L_{1}$ at $C_{g}=0.6$ in the $X$ direction. (b) Stress-strain comparison curves of the unimodal and bimodal polymer-grafted NPs; hollow represents unimodal systems and solid represents bimodal systems. The red, blue and magenta curves represent systems A, B and C, respectively. (c) The non-bonded interaction of matrix-grafted chains of unimodal and bimodal systems in system $C$ during the uniaxial deformation.
NPs are well dispersed at $L_{1}=25$. With the increase of $L_{1}$, the dispersion of NPs gets worse, and NPs aggregate to form clusters. The radial distribution function (RDF) between NPs was also characterized. As shown in Fig. 6(b), a sharp peak in the RDF appears at approximately $r=4 \sigma$ except for $L_{1}=25$, denoting the direct contact of NPs. With the increase of $L_{\mathrm{l}}$, this peak gradually increases. With the increase of $L_{1}$ at a constant content of grafted beads, the decrease of $\Sigma$ will result in a decrease of the spatial repulsion of the grafted chains, which is consistent with the NP clustering, as shown in Fig. 6(a).

We have investigated the effect of $L_{1}$ on the mechanical properties, and a uniaxial tension in the $X$-direction was applied. The tensile stress-strain behavior and the bond orientation during the uniaxial tensile deformation are shown in Fig. 7(a) and Fig. S4 (ESI $\dagger$ ). We use the second-order Legendre polynomials, $\left\langle P_{2}(\cos \theta)\right\rangle$, to express the bond orientation. This function describes the average of the $\theta$ angle between a given bond vector and the reference (stretching) direction,

$$
\left\langle P_{2}(\cos \theta)\right\rangle=\left(3\left\langle\cos ^{2} \theta\right\rangle\right) / 2
$$

The values of $\left\langle P_{2}(\cos \theta)\right\rangle$ range from -0.5 to $1 ;-0.5,1$ and 0 indicate a perfect orientation perpendicular to the reference direction, parallel to the reference direction, or randomly oriented, respectively. Fig. 7(a) shows that the tensile stress curves are strikingly similar for different values of $L_{1}$. In Fig. S4 (ESI $\dagger$ ), we observe that during the uniaxial deformation, the bond orientations of matrix chains, grafted long chains and grafted short chains are almost identical, which can explain the similarity of the stress-strain response. Therefore, we think that the mechanical properties of the systems are closely related to $n_{\mathrm{g}}$.

To explore further the relationship between the mechanical properties of the system and the length of grafted chains, we fix $\Sigma=0.4$ and $n_{g}$, to compare the tensile properties of the unimodal and bimodal systems. Here, we set up three sets of comparison tests, and the details of the system composition are shown in Table 4. Then, we compare their mechanical properties, the red, blue and magenta curves representing systems A, B and C, respectively. From Fig. 7(b), we can see that the curves of the three groups of comparison experiments are close to coincidence. That is to say, the unimodal system and the bimodal system have similar mechanical properties.

Table 4 Parameters of each simulated system with unimodal and bimodal polymer-grafted $\mathrm{NPs}^{\mathrm{a}}$

\begin{tabular}{llllllll}
\hline & \multicolumn{3}{c}{ Unimodal system } & & \multicolumn{3}{c}{ Bimodal system } \\
\cline { 2 - 4 } System & $L_{\mathrm{g}}$ & $N_{\mathrm{g}}$ & $n_{\mathrm{g}}$ & & $L_{\mathrm{l}} / L_{\mathrm{s}}$ & $N_{\mathrm{l}} / N_{\mathrm{s}}$ & $n_{\mathrm{g}}$ \\
\hline $\mathrm{A}$ & 25 & 20 & 15000 & & $40 / 10$ & $10 / 10$ & 15000 \\
$\mathrm{~B}$ & 38 & 20 & 22800 & & $50 / 25$ & $10 / 10$ & 22500 \\
$\mathrm{C}$ & 50 & 20 & 30000 & & $75 / 25$ & $10 / 10$ & 30000
\end{tabular}

${ }^{a}$ In the unimodal system, $L_{\mathrm{g}}$ - length of grafted chains, and $N_{\mathrm{g}}$ number of grafted chains per NP. In the bimodal system, $L_{1}-$ length of grafted long chains, $L_{\mathrm{s}}$ - length of grafted short chains, $N_{\mathrm{l}}$ - number of grafted long chains per NP, and $N_{\mathrm{s}}$ - number of grafted short chains per NP. $n_{\mathrm{g}}$ - total number of grafted beads. 
Next, taking system $\mathrm{C}$ as an example, we explore the interface effect of the unimodal and bimodal systems in the stretching process. Although in the bimodal system, the non-bonded interaction of the matrix-grafted long chains is much higher than that of the matrix-grafted short chains, the total non-bonded interaction of the matrix-grafted chains (magenta solid line in Fig. 7(c)) is strikingly consistent with that in the unimodal system (magenta dotted line), indicating that the contribution of long-short chains to the interface interaction is the same as that of the homogeneous chains. This also further confirms our statement that the
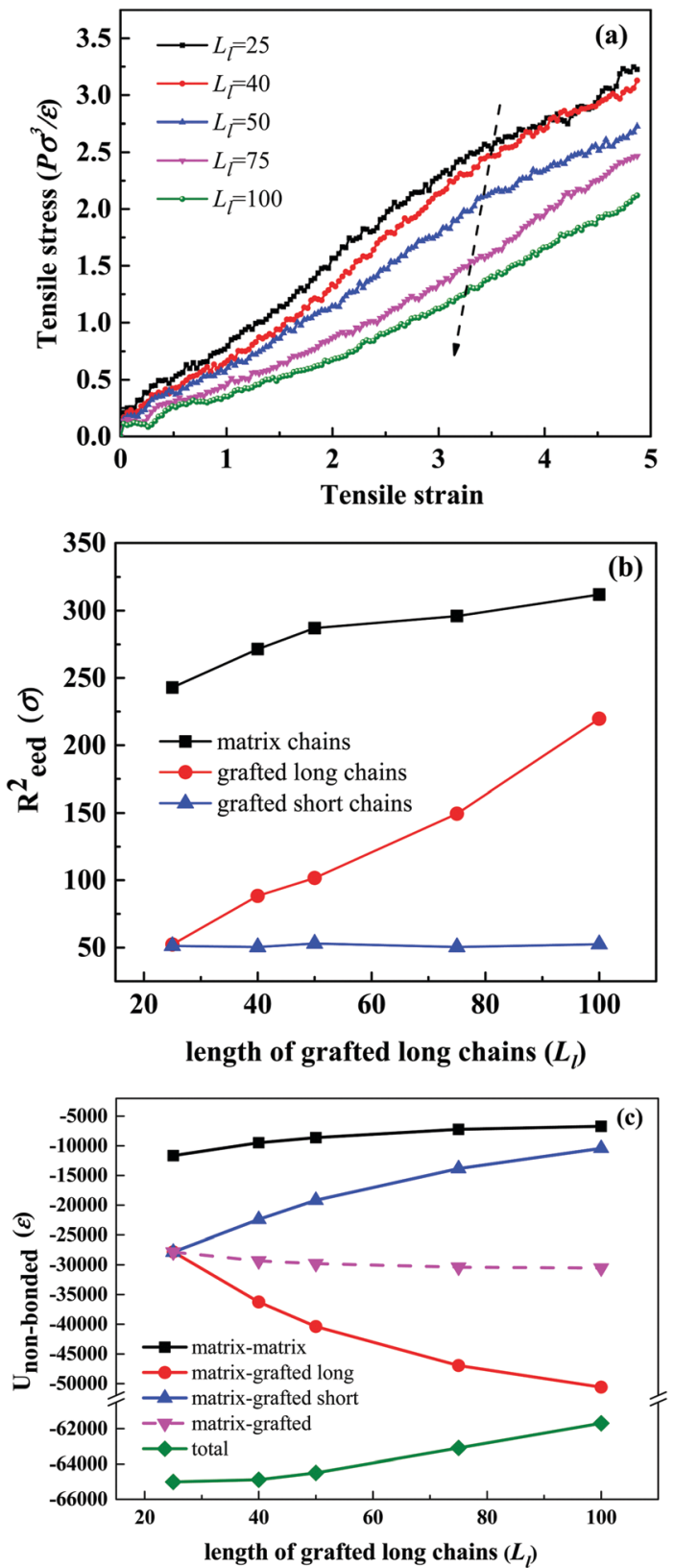

Fig. 8 The effect of the length of grafted long chains $L_{\mathrm{l}}$ at increasing $C_{\mathrm{g}}$ on (a) the tensile stress-strain behavior in the $X$ direction, (b) the meansquared end-to-end distance $R_{\text {eed }}{ }^{2}$ of the matrix chains, grafted long chains and grafted short chains in the equilibrium state, and (c) the nonbonded interaction of various parts of the system in the equilibrium state. mechanical properties of systems are closely related to $n_{\mathrm{g}}$, or the synergistic effect between the length and density of grafted chains leads to no significant difference in the performance of systems.

3.2.2 Effect of bimodal distribution of grafted chains with increasing $C_{\mathbf{g}}$ (from 0.6 to $\mathbf{0 . 7 9}$ ). We continue to explore the effect of different $L_{1}$ on the dispersion of NPs and mechanical properties of the systems, in which we fix $\Sigma=0.4$ and $L_{\mathrm{s}}=25$; more details are listed in Table 3. Similar to the above study, we study the dispersion of NPs first, and more detailed analysis is shown S5 (ESI $\dagger$ ). The brush configuration is also characterized by Fig. S6 (ESI $\dagger$ ). Then, we investigate how the end-grafting influences the mechanical properties of PNCs, which is shown in Fig. 8(a). It can be observed that the tensile stress at any strain decreases monotonically with the increase of $L_{\mathrm{l}}$.

To understand further the difference in this behavior, we calculate the mean-squared end-to-end distance $R_{\text {eed }}^{2}$ of the matrix chains, grafted long chains and grafted short chains as a function of $L_{1}$ in the equilibrium state. We can easily observe from Fig. 8(b) that $R_{\text {eed }}{ }^{2}$ of the matrix chains and the grafted long chains increases gradually with the increase of $L_{1}$, which means that longer grafted chains lead to a lower density and a larger size of the polymer network. As we all know, the expansion of the system may reduce the non-bonded interaction of the whole system, so the specific information about the non-bonded interaction is shown in Fig. 8(c). Clearly, the
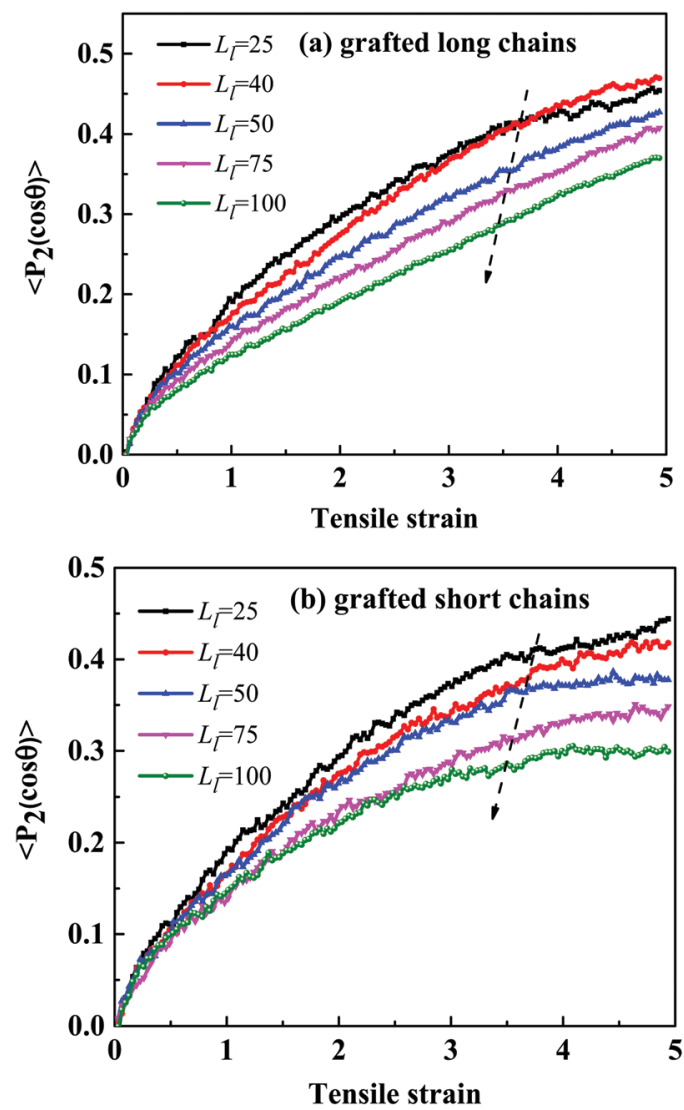

Fig. 9 The effect of grafted long chain length $L_{1}$ on the bond orientation of the grafted long chains (a) and the grafted short chains (b) of the systems during the uniaxial deformation. 
non-bonded interaction energy of the matrix-grafted brush increases slightly with the increase of $L_{1}$ and the non-bonded interaction energy of the matrix chains becomes weaker. In other words, improving $L_{1}$ will lead to the expansion of the grafted chains and the matrix chains, which is consistent with the above discussion. What's more, the total non-bonded interaction energy becomes weaker with the increase of $L_{\mathrm{l}}$, meaning pulling the entangled polymer chains needs less tensile force, which may result in a decrease of the tensile stress.

By analyzing the bond orientation behavior of each part during the uniaxial deformation, we find that the orientation degrees, both of the grafted long and short chains, decrease with the increase of $L_{1}$ throughout the deformation process, as shown in Fig. 9. The curves of the matrix chains are very similar in Fig. S7 (ESI $\dagger$ ), indicating that the increase of $L_{1}$ is not conducive to the bond orientation of grafted chains. We infer that the stress differences in the five systems with different $L_{1}$ are weakly affected by the bond orientation of the matrix chains, and may strongly depend on the decrease of the total non-bonded interaction energy of the system and the bond orientation of the grafted chains.

\section{Conclusions}

In this study, we adopt coarse-grained molecular-dynamics simulations in order to investigate the effects of the structure and the bimodal dispersion of grafted polymer chains on the mechanical properties of the resulting PNCs. For the mechanically interlocked PNCs, we have successfully constructed an ideal mechanically interlocked structure by using two different methods, and demonstrated their remarkable mechanical reinforcement effect. One method is to change the stiffness of the matrix chains during the equilibration process. In the first equilibration step, the stiffness promotes matrix chains to thread through the grafted rings, and in the second equilibration step, their flexibility induces the locking of the resulting structure. The mechanical enhancement effect, above $200 \%$ on average, is the largest at the bending constant $k=20.0$ in the first equilibration step. The second method is to change the size and the shape of the grafted rings by adjusting the equilibrium value of the corresponding bending angle $\theta_{0}$. For $\theta_{0}=120^{\circ}$ in the grafted rings and for $k=5.0$ in the matrix chains, the reinforcement effect, above $700 \%$, is the most remarkable in all the simulated systems.

The mechanical properties of the composites with the bimodal polymer-grafted NPs have been analyzed. The stressstrain curves exhibit striking similarity when the fraction $C_{\mathrm{g}}$ of the grafted chains is fixed, indicating that the mechanical properties of the systems are closely related to the total number of beads of the grafted chains. Furthermore, when $C_{\mathrm{g}}$ increases with the increase of $L_{\mathrm{l}}$, keeping the grafting density unchanged, the mechanical properties decrease monotonically. This result is attributed to the decrease of the total non-bonded interaction energy of the system in the equilibration state and to the reduced bond orientation of both the long and short grafted chains during the uniaxial deformation. In general, it is anticipated that our study could provide some guidance on tailoring the mechanical performance of grafted NP-filled polymer nanocomposites, by taking advantage of varying the structure and length of the grafted polymer chains.

\section{Conflicts of interest}

There are no conflicts to declare.

\section{Acknowledgements}

We acknowledge the financial support from the Major Program (51790502) of the National Nature Science Foundation of China, the National Natural Science Foundation of China (51873006 and 21674010), the Beijing Natural Science Foundation (2182053) and the National 973 Basic Research Program of China 2015CB654700 (2015CB654704). The super-calculation center of "Tianhe number two" and the cloud calculation platform of Beijing University of Chemical Technology (BUCT) are both greatly appreciated.

\section{References}

1 V. M. Svetlichnyi, K. A. Romashkova, L. I. Subbotina, V. E. Yudin, E. V. Popova, I. V. Gofman, T. E. Sukhanova, E. N. Vlasova and N. V. Afanas'eva, Russ. J. Appl. Chem., 2013, 86, 415-422.

2 M. R. Bockstaller and E. L. Thomas, Phys. Rev. Lett., 2004, 93, 166106.

3 J. Bae, E. Glogowski, S. Gupta, W. Chen, T. Emrick and T. P. Russell, Macromolecules, 2008, 41, 2722-2726.

4 C. G. Martins, N. M. Larocca, D. R. Paul and L. A. Pessan, Polymer, 2009, 50, 1743-1754.

5 S. S. Rekhviashvili, E. V. Kishtikova and B. A. Rozenberg, Russ. J. Phys. Chem. B, 2009, 3, 1008-1014.

6 B. Natarajan, T. Neely, A. Rungta, B. C. Benicewicz and L. S. Schadler, Macromolecules, 2013, 46, 4909-4918.

7 S. Lee, B. Lee, B. J. Kim, J. Park, M. Yoo, W. K. Bae, K. Char, C. J. Hawker, J. Bang and J. Cho, J. Am. Chem. Soc., 2009, 131, 2579-2587.

8 Y. Kim, C. Lee, I. Shim, D. Wang and J. Cho, Adv. Mater., 2010, 22, 5140-5144.

9 J. Gu, Y. Yan, Y. S. Zhao and J. Yao, Adv. Mater., 2012, 24, 2249-2253.

10 M. Sureshkumar, D. Y. Siswanto and C.-K. Lee, J. Mater. Chem., 2010, 20, 6948-6955.

11 V. Ganesan and A. Jayaraman, Soft Matter, 2014, 10, 13-38. 12 A. Hashemi, N. Jouault, G. A. Williams, D. Zhao, K. J. Cheng, J. W. Kysar, Z. Guan and S. K. Kumart, Nano Lett., 2015, 15, 5465-5471.

13 C. Chevigny, N. Jouault, F. Dalmas, F. Boue and J. Jestin, J. Polym. Sci., Part B: Polym. Phys., 2011, 49, 781-791.

14 H. S. Wang, A. Khan, Y. Choe, J. Huh and J. Bang, Macromolecules, 2017, 50, 5025-5032. 
15 J. Shen, J. Liu, Y. Gao, X. Li and L. Zhang, Soft Matter, 2014, 10, 5099-5113.

16 J. Shen, J. Liu, H. Li, Y. Gao, X. Li, Y. Wu and L. Zhang, Phys. Chem. Chem. Phys., 2015, 17, 7196-7207.

17 J. Liu, S. Wu, L. Zhang, W. Wang and D. Cao, Phys. Chem. Chem. Phys., 2011, 13, 518-529.

18 P. Akcora, H. Liu, S. K. Kumar, J. Moll, Y. Li, B. C. Benicewicz, L. S. Schadler, D. Acehan, A. Z. Panagiotopoulos, V. Pryamitsyn, V. Ganesan, J. Ilavsky, P. Thiyagarajan, R. H. Colby and J. F. Douglas, Nat. Mater., 2009, 8, 354-359.

19 X. L. Dou, X. L. Liu, Y. Zhang, H. Feng, J. F. Chen and S. Du, Appl. Phys. Lett., 2009, 95, 306.

20 P. Akcora, S. K. Kumar, J. Moll, S. Lewis, L. S. Schadler, Y. Li, B. C. Benicewicz, A. Sandy, S. Narayanan, J. Illavsky, P. Thiyagarajan, R. H. Colby and J. F. Douglas, Macromolecules, 2010, 43, 1003-1010.

21 J. F. Moll, P. Akcora, A. Rungta, S. Gong, R. H. Colby, B. C. Benicewicz and S. K. Kumar, Macromolecules, 2011, 44, 7473-7477.

22 S. K. Kumar, V. Ganesan and R. A. Riggleman, J. Chem. Phys., 2017, 147, 020901.

23 N. Molinari, A. P. Sutton and A. A. Mostofi, Phys. Chem. Chem. Phys., 2018, 20, 23085-23094.

24 J. U. Kim and M. W. Matsen, Phys. Rev. Lett., 2009, 102, 078303. 25 S. Kim, M. Yoo, N. Kang, B. Moon, B. J. Kim, S.-H. Choi, J. U. Kim and J. Bang, ACS Appl. Mater. Interfaces, 2013, 5, 5659-5666.

26 Y. Li, T. M. Krentz, L. Wang, B. C. Benicewicz and L. S. Schadler, ACS Appl. Mater. Interfaces, 2014, 6, 6005-6021.

27 D. L. Ramasamy, V. Puhakka, B. Doshi, S. Iftekhar and M. Sillanpaa, Chem. Eng. J., 2019, 365, 291-304.

28 X. D. Qian, Polym. Compos., 2018, 39, 4637-4645.

29 R. Yuan, J. Yuan, Y. P. Wu, P. F. Ju, L. Ji, H. X. Li, L. Chen, H. D. Zhou and J. M. Chen, J. Mater. Sci., 2018, 53, 11460-11472.

30 A. Alam, M. D. H. Beg, R. M. Yunus, M. Bijarimi, M. F. Mina, K. H. Maria and T. Mieno, Polym. Test., 2018, 68, 116-125.

31 M. Kilbey, D. Street, R. Ramirez, J. Sangoro and J. Messman, Abstr. Pap. Am. Chem. Soc., 2018, 255, 2.

32 P. R. Ye, P. F. Cao, Q. Y. Chen and R. Advincula, Macromol. Mater. Eng., 2019, 304, 10.

33 A. Tibbits, K. Park, J. Streit, L. Drummy and R. Vaia, Abstr. Pap. Am. Chem. Soc., 2018, 255, 1.

34 X. Wang, S. Y. Jing, Y. Y. Liu, X. M. Qiu and Y. B. Tan, RSC Adv., 2017, 7, 13112-13122.

35 S. K. Kumar, N. Jouault, B. Benicewicz and T. Neely, Macromolecules, 2013, 46, 3199-3214.

36 J. R. Brown, Y. Seo, T. A. Maula and L. M. Hall, J. Chem. Phys., 2016, 144, 124904.

37 D. Maillard, S. K. Kumar, A. Rungta, B. C. Benicewicz and R. E. Prud'homme, Nano Lett., 2011, 11, 4569-4573.

38 J. Moll, S. K. Kumar, F. Snijkers, D. Vlassopoulos, A. Rungta, B. C. Benicewicz, E. Gomez, J. Ilavsky and R. H. Colby, ACS Macro Lett., 2013, 2, 1051-1055.
39 J. Shen, J. Liu, Y. Gao, D. Cao and L. Zhang, Langmuir, 2011, 27, 15213-15222.

40 D. M. Trombly and V. Ganesan, J. Chem. Phys., 2010, 133, 154904.

41 P. F. Green, Soft Matter, 2011, 7, 7914-7926.

42 S. Srivastava, P. Agarwal and L. A. Archer, Langmuir, 2012, 28, 6276-6281.

43 J. Liu, Y. Gao, D. Cao, L. Zhang and Z. Guo, Langmuir, 2011, 27, 7926-7933.

44 J. Liu, Z. Wang, Z. Zhang, J. Shen, Y. Chen, Z. Zheng, L. Zhang and A. V. Lyulin, J. Phys. Chem. B, 2017, 121, 9311-9318.

45 N. Suzuki, M. Ito and F. Yatsuyanagi, Polymer, 2005, 46, 193-201.

46 J. H. Chen, M. Z. Rong, W. H. Ruan and M. Q. Zhang, Compos. Sci. Technol., 2009, 69, 252-259.

47 M. Z. Rong, M. Q. Zhang, Y. X. Zheng, H. M. Zeng, R. Walter and K. Friedrich, Polymer, 2001, 42, 167-183.

48 S. Goyal and F. A. Escobedo, J. Chem. Phys., 2011, 135, 184902.

49 M. Gauthier and A. Munam, Polymer, 2009, 50, 6032-6042.

50 S. K. Kumar and R. Krishnamoorti, Annu. Rev. Chem. Biomol. Eng., 2010, 1, 37-58.

51 Z. Wang, Z. Zheng, J. Liu, Y. Wu and L. Zhang, Polymers, 2016, 8, 270.

52 V. V. Ginzburg, Macromolecules, 2013, 46, 9798-9805.

53 A. Lopez-Moreno, B. Nieto-Ortega, M. Moffa, A. de Juan, M. M. Bernal, J. P. Fernandez-Blazquez, J. J. Vilatela, D. Pisignano and E. M. Perez, ACS Nano, 2016, 10, 8012-8018.

54 E. M. Perez, Chem. - Eur. J., 2017, 23, 12681-12689.

55 Y. Li, P. Tao, A. Viswanath, B. C. Benicewicz and L. S. Schadler, Langmuir, 2013, 29, 1211-1220.

56 A. Rungta, B. Natarajan, T. Neely, D. Dukes, L. S. Schadler and B. C. Benicewicz, Macromolecules, 2012, 45, 9303-9311.

57 R. Shi, H.-J. Qian and Z.-Y. Lu, Phys. Chem. Chem. Phys., 2017, 19, 16524-16532.

58 Z. Shen, L. Zhang, J. Cao, J. Tian, L. Liu, G. Wang, Z. Zhao, X. Wang, R. Zhang and S. Liu, J. Environ. Monit., 2012, 14, 3000-3008.

59 K. Kremer and G. S. Grest, J. Chem. Phys., 1991, 94, 4103.

60 N. Goga, M. N. Melo, A. J. Rzepiela, A. H. de Vries, A. Hadar, S. J. Marrink and H. J. C. Berendsen, J. Chem. Theory Comput., 2015, 11, 1389-1398.

61 M. K. Crawford, R. J. Smalley, G. Cohen, B. Hogan, B. Wood, S. K. Kumar, Y. B. Melnichenko, L. He, W. Guise and B. Hammouda, Phys. Rev. Lett., 2013, 110, 196001.

62 S. N. Mohammad, Nanotechnology, 2012, 23, 285707.

63 M. Kroger and S. Hess, Phys. Rev. Lett., 2000, 85, 1128-1131.

64 G. N. Toepperwein, N. C. Karayiannis, R. A. Riggleman, M. Kroeger and J. J. de Pablo, Macromolecules, 2011, 44, 1034-1045.

65 G. Srinivas, D. E. Discher and M. L. Klein, Nat. Mater., 2004, 3, 638-644.

66 G. Raos, M. Moreno and S. Elli, Macromolecules, 2006, 39, 6744-6751. 
67 B. Ensing, S. O. Nielsen, P. B. Moore, M. L. Klein and M. Parrinello, J. Chem. Theory Comput., 2007, 3, 1100-1105.

68 Y. Zhang, P. Tiwary, J. S. Parent, M. Kontopoulou and C. B. Park, Polymer, 2013, 54, 4814-4819.

69 G. Hou, X. Xia, J. Liu, W. Wang, M. Dong and L. Zhang, J. Phys. Chem. B, 2019, 123, 2157-2168.

70 G. Buck and E. J. Rawdon, Phys. Rev. E: Stat., Nonlinear, Soft Matter Phys., 2004, 70, 011803.

71 R. Everaers, S. K. Sukumaran, G. S. Grest, C. Svaneborg, A. Sivasubramanian and K. Kremer, Science, 2004, 303, 823-826.
72 S. K. Sukumaran, G. S. Grest, K. Kremer and R. Everaers, J. Polym. Sci., Part B: Polym. Phys., 2005, 43, 917-933.

73 J. Liu, Z. Zheng, F. Li, W. Lei, Y. Gao, Y. Wu, L. Zhang and Z. L. Wang, Nano Energy, 2016, 28, 87-96.

74 W. Wang, G. Hou, Z. Zheng, L. Wang, J. Liu, Y. Wu, L. Zhang and A. V. Lyulin, Phys. Chem. Chem. Phys., 2017, 19, 15808-15820.

75 P. Norouzzadeh, A. Nozariasbmarz, J. S. Krasinski and D. Vashaee, J. Appl. Phys., 2015, 117, 214303.

76 J. Liu, J. X. Shen, D. P. Cao and L. Q. Zhang, Acta Polym. Sin., 2016, 8, 1048-1061. 